\title{
Specific role of JNK in the maintenance of the tumor-initiating capacity of A549 human non-small cell lung cancer cells
}

\author{
MASASHI OKADA ${ }^{1}$, KEITA SHIBUYA ${ }^{1,5,6}$, ATSUSHI SATO ${ }^{1,2}$, SHIZUKA SEINO ${ }^{1,5,6}$, ERIKO WATANABE ${ }^{1,5,6}$, \\ SHUHEI SUZUKI $^{1,3}$, MANABU SEINO ${ }^{1,4}$ and CHIFUMI KITANAKA ${ }^{1,5,6}$

\begin{abstract}
Departments of ${ }^{1}$ Molecular Cancer Science, ${ }^{2}$ Neurosurgery, ${ }^{3}$ Clinical Oncology and ${ }^{4}$ Obstetrics and Gynecology, Yamagata University School of Medicine, Yamagata 990-9585; ${ }^{5}$ Oncology Research Center, Research Institute for Advanced Molecular Epidemiology, Yamagata University, Yamagata 990-9585;

${ }^{6}$ Global COE program for Medical Sciences, Japan Society for Promotion of Science, Tokyo 102-8471, Japan
\end{abstract}

Received May 16, 2013; Accepted June 28, 2013

DOI: $10.3892 /$ or.2013.2655

\begin{abstract}
Deregulation of c-Jun $\mathrm{NH}_{2}$-terminal kinase (JNK) signaling is now increasingly reported in a variety of human malignancies. Non-small cell lung cancer (NSCLC) is among such human malignancies with aberrant JNK activation; yet the exact role(s) of JNK deregulation in NSCLC biology, in particular in vivo, remains unclear. Here, we demonstrated a specific role of JNK in the control of the tumor-initiating capacity of A549 cells derived from human lung adenocarcinoma, a major subtype of NSCLC. Despite its potent inhibitory activity on A549 cell growth in vitro, SP600125, a reversible JNK inhibitor, failed to inhibit the growth of pre-established A549 xenografts in vivo when systemically administered. Nevertheless, the same SP600125 treatment caused a marked reduction in the tumor-initiating population within the A549 tumors, suggesting that JNK may be specifically required in vivo for the maintenance of the tumor-initiating population of tumor cells rather than for proliferation and survival of the entire cell population. Furthermore, A549 cells either pretreated with SP600125 or transiently transfected with siRNAs against the JNK genes in vitro showed substantially reduced ability to initiate tumor formation upon implantation into nude mice, implying that the cell intrinsic JNK activity of A549 cells is essential for the maintenance of their tumor-initiating capacity. To our knowledge, this is the first demonstration that JNK is involved in the control of the tumor-initiating capacity of NSCLC cells. Our findings also give rise to an intriguing possibility that therapies targeting JNK could contribute to prevention of relapse and/or metastasis of NSCLC through elimination of tumor-initiating cells.
\end{abstract}

Correspondence to: Professor Chifumi Kitanaka, Department of Molecular Cancer Science, Yamagata University School of Medicine, 2-2-2 Iida-nishi, Yamagata 990-9585, Japan

E-mail: ckitanak@med.id.yamagata-u.ac.jp

Key words: xenograft analysis, tumorigenicity, lung cancer, JNK, cancer stem cell

\section{Introduction}

The c-Jun $\mathrm{NH}_{2}$-terminal kinases (JNKs) belong to a subfamily of mitogen-activated protein kinases implicated in the transduction of a wide array of intracellular signals associated with, for instance, cellular proliferation, differentiation, development, the inflammatory response and apoptosis $(1,2)$. Reflecting such versatile functions of JNK in signal transduction, a growing body of evidence now suggests that JNKs may also play a significant role in the biology of human cancers (3-5). Indeed, aberrant activation of JNK has been increasingly reported in a variety of human cancers, and the roles of JNK in the control of fundamental cellular properties of cancer cells, such as proliferation, survival and migration, have been documented (2-5). Significantly, adding to such roles of JNK in cancer cells in general, we and others have recently identified a specific role of JNK in the control of cancer stem cells (CSCs) of glioblastoma (5-7), a particular subpopulation of cancer cells now presumed to have a pivotal role in cancer relapse and/or metastasis as well as in therapy resistance $(8,9)$. Most importantly, we demonstrated for the first time in our recent study that 'sustained' JNK activity is not only required for the prevention of differentiation and maintenance of the 'stem cell state' of glioblastoma CSCs but also for the maintenance of their 'tumor-initiating capacity'. Furthermore, we showed in the study that transient, short-term inhibition of JNK in vivo is sufficient to exert a long-lasting suppressive effect on the tumor-initiating capacity of glioblastoma cells, suggesting that JNK could make an excellent therapeutic target to achieve long-term control of glioblastoma, one of the deadliest of all human cancers $(5,6)$. However, to date, it remains totally unknown whether such a role of JNK in the control of the stemness and/or the tumor-initiating capacity of tumor cells is unique to glioblastoma or is commonly shared by other human cancers.

Lung cancer is currently the leading cause of cancer-related mortality in the world (10), with non-small cell lung cancer (NSCLC) accounting for the majority of lung cancer cases ( $>0 \%)(10,11)$. Similarly to glioblastomas (12-15), aberrant activation of JNK has been observed frequently in NSCLC tumors $(16,17)$, and a number of studies do provide evidence 
supporting the idea that JNK has a positive role in promoting the growth of NSCLC tumors (16-18). Nevertheless, evidence is not yet sufficient, with the exact role(s) of JNK in NSCLC cells, in particular in vivo, remaining poorly understood. Here in this study, therefore, we investigated the role of JNK in NSCLC using a human NSCLC cell line A549 (19). Intriguingly, the results of the xenograft analyses of the present study demonstrated that JNK is more specifically involved in tumor initiation than in bulk tumor growth (i.e., growth of already established tumors) of A549 cells. Our findings thus suggest that the maintenance of the tumor-initiating population may be one of the primary roles of JNK in NSCLC tumors in vivo and that such a role of $\mathrm{JNK}$ is shared by human cancers other than glioblastoma.

\section{Materials and methods}

Antibodies and reagents. SP600125 was purchased from Calbiochem (La Jolla, CA, USA) and was dissolved in dimethylsulfoxide (DMSO) to prepare a $50 \mathrm{mM}$ stock solution. Anti-c-Jun (\#9165) and anti-phospho-c-Jun (Ser63) (\#9261) antibodies were purchased from Cell Signaling Technology, Inc. (Beverly, MA, USA). Anti- $\beta$-actin (A1978) was from Sigma (St. Louis, MO, USA). Anti-JNK1 (sc-474) and anti-JNK2 (sc-7345) were from Santa Cruz Biotechnology, Inc. (Santa Cruz, CA, USA).

Cell culture. The human NSCLC cell line A549 was obtained from the Riken BioResource Center. To verify the authenticity of the A549 cells used in this study, the A549 cells in actual use were subjected to genotyping by short tandem repeat (STR) profiling (Bio-Synthesis, Inc., Lewisville, TX, USA) at the completion of this study. For the serum-free culture of A549, a culture condition reported to allow the selection of undifferentiated cancer stem cells including those of lung cancer (20-22), cells were cultured on collagen-I-coated dishes (IWAKI) in serum-free culture medium [Dulbecco's modified Eagle's medium (DMEM)/F12 supplemented with B27 (both from Gibco-BRL, Carlsbad, CA, USA)], 20 ng/ml EGF, $20 \mathrm{ng} /$ ml FGF2 (Peprotech, Inc., Rocky Hill, NJ, USA), D-(+)-glucose (final concentration, $26.2 \mathrm{mM}$ ), $2 \mathrm{mM}$ L-glutamine (final concentration, $4.5 \mathrm{mM}$ ), 100 units/ml penicillin, and $100 \mu \mathrm{g} / \mathrm{ml}$ streptomycin). The serum-free culture medium was changed every 3 days, and EGF and FGF2 were added to the culture medium every day. For the serum culture of A549 cells, cells were maintained in DMEM/F12 with $10 \%$ fetal bovine serum and antibiotics (100 units/ml penicillin, $100 \mu \mathrm{g} /$ $\mathrm{ml}$ streptomycin). Unless otherwise indicated, A549 cells were maintained and subjected to experiments using the serum-free culture condition. Throughout the study, the cell number was determined using a hemocytometer, and the cell viability was examined by the dye exclusion method ( $0.2 \%$ trypan blue). Cell viability (\%) was defined as $100 \mathrm{x}$ 'the number of viable cells'/ ('the number of viable cells' + 'the number of dead cells').

Gene silencing by siRNA. siRNAs against human JNK1 (HSS108547), JNK2 (HSS108550), and Stealth RNAi ${ }^{\mathrm{TM}}$ siRNA negative control duplexes (Medium GC Duplexes \#2) were purchased from Invitrogen Life Technologies (Carlsbad, CA, USA). Transfection of siRNAs was performed using Lipofectamine RNAiMAX (Invitrogen Life Technologies) according to the manufacturer's instructions. To achieve sustained knockdown of the target genes, siRNA transfection was repeated 4 days after the initial transfection.

Immunoblot analysis. Cells were washed with ice-cold phosphate-buffered saline (PBS) and lysed in the RIPA buffer $[10 \mathrm{mM}$ Tris-HCl (pH 7.4), 0.1\% SDS, $1 \%$ sodium deoxycholate, $150 \mathrm{mM} \mathrm{NaCl}, 1 \mathrm{mM}$ EDTA, $1.5 \mathrm{mM} \mathrm{Na} \mathrm{VO}_{4}$, $10 \mathrm{mM} \mathrm{NaF}, 10 \mathrm{mM}$ sodium pyrophosphate, $10 \mathrm{mM}$ sodium $\beta$-glycerophosphate and $1 \%$ protease inhibitor cocktail set III (Calbiochem)]. After centrifugation for $10 \mathrm{~min}$ at $14,000 \mathrm{x} \mathrm{g}$ at $4^{\circ} \mathrm{C}$, the supernatants were recovered as the cell lysates, and the protein concentration of the cell lysates was determined by the BCA protein assay kit (Pierce Biotechnology, Inc., Rockford, IL, USA). Cell lysates containing equal amounts of protein were separated by SDS-PAGE and transferred to a polyvinylidene difluoride membrane. The membrane was probed with a primary antibody and then with an appropriate HRP-conjugated secondary antibody according to the protocol recommended by the manufacturer of each antibody. Immunoreactive bands were visualized using Immobilon Western Chemiluminescent HRP Substrate (Millipore, Billerica, MA, USA).

Mouse studies. Mouse xenograft studies were carried out essentially as previously described $(6,23)$. For subcutaneous implantation of A549 cells, cells were suspended in $200 \mu \mathrm{l}$ of PBS and injected into the flank region of 5- to 7-week-old male BALB/cAJcl-nu/nu mice (Clea Japan, Inc.). After implantation, the recipient mice were monitored for general health status and the presence of subcutaneous tumors. Tumor volume was determined by measuring tumor diameters (measurement of 2 perpendicular axes of tumors) using a caliper and calculated as $1 / 2 \times$ (larger diameter) $\mathrm{x}$ (smaller diameter) ${ }^{2}$. For serial transplantation, excised tumors were washed in chilled sterile PBS and transferred into DMEM/F12, minced with scissors, and incubated in Accutase for $30 \mathrm{~min}$ at $37^{\circ} \mathrm{C}$. After washing with $\mathrm{DMEM} / \mathrm{F} 12$, the cells were resuspended in DMEM/F12 and filtered through a $70-\mu \mathrm{m}$ strainer. After determination of cell number and viability, the single-cell suspension of the tumor cells was subjected to subcutaneous injection. For systemic administration of SP600125, the SP600125 stock solution (50 mM in DMSO) was diluted in PBS to prepare $200 \mu 1$ solutions of SP600125 for each injection. The SP600125 solutions were injected intraperitoneally into nude mice. Control mice received $200 \mu \mathrm{l}$ of DMSO diluted in PBS. Note that all the control- and SP600125-treated mice received an equal volume of DMSO per body weight ( $3.6 \mathrm{ml} / \mathrm{kg} / \mathrm{day})$. All animal experiments were performed under a protocol approved by the Animal Research Committee of Yamagata University.

Statistical analysis. Results are expressed as the means \pm SD, and differences were compared using the 2-tailed Student's t-test. P-values $<0.05$ were considered statistically significant and indicated with asterisks (*) in the figures.

\section{Results}

Rapid and potent inhibition of A549 cell proliferation by JNK inhibition in vitro. To examine the role of JNK in A549 

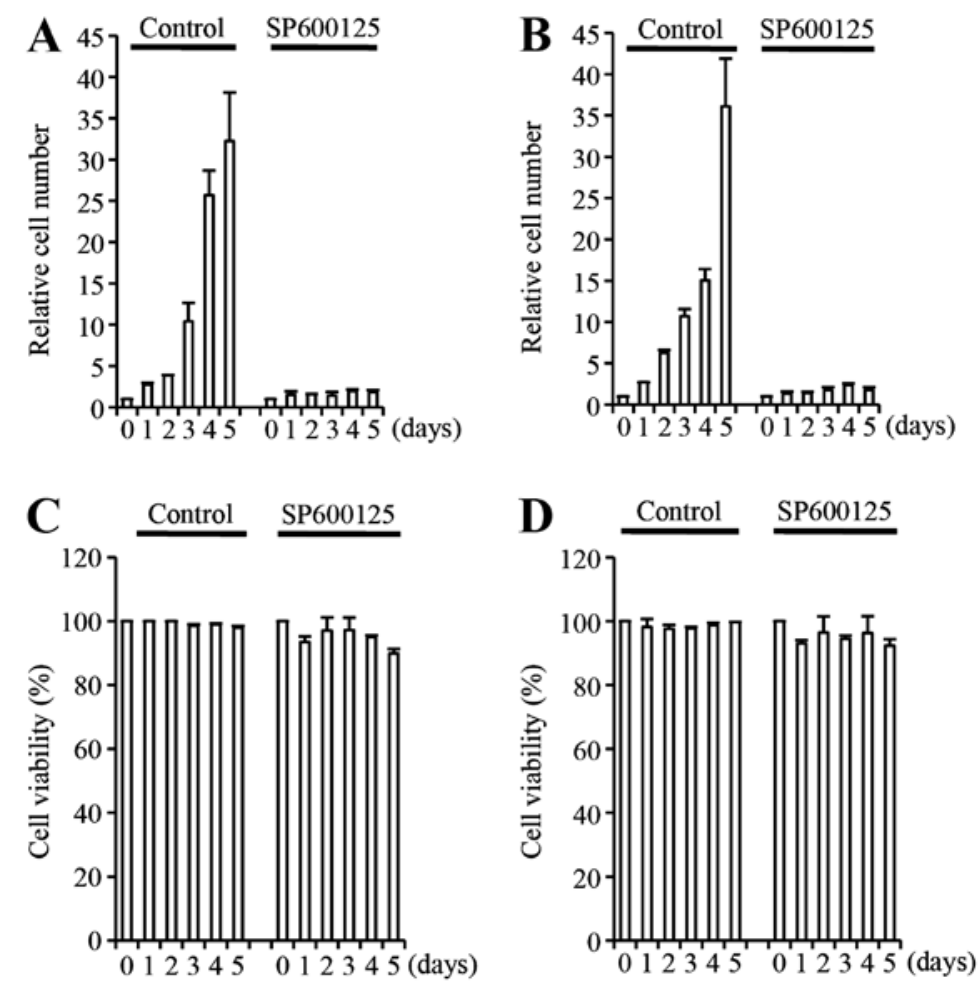

Figure 1. Inhibition of A549 cell growth by SP600125 in vitro. A549 cells maintained in the presence (A and C) or absence (B and D) of serum were treated without (control) or with $20 \mu \mathrm{M}$ SP600125. The number of viable cells (A and B) and the cellular viability (C and D) at each time point were determined. In $\mathrm{A}$ and $\mathrm{B}$, the data are expressed as the relative number of viable cells compared to that of day 0 (determined just prior to SP600125 treatment), which was arbitrarily set to 1 . In $A-D$, values represent the mean \pm SD of 3 independent experiments.

cells, we first tested the effect of JNK inhibition on A549 cell growth in vitro using SP600125, an ATP-competitive, reversible inhibitor of JNK (24). When A549 cells cultured in the presence of serum were treated with SP600125 at $20 \mu \mathrm{M}$, the JNK inhibitor caused a pronounced proliferation block on A549 cells without causing significant toxicity (Fig. 1A and C). Growth suppression by SP600125 was rapid and potent, being apparent as early as 1 day after the initiation of the inhibitor treatment and was already marked by 3 days of inhibitor treatment (Fig. 1A). Identical experiments were conducted with A549 cells maintained in serum-free culture condition, reported to allow selective expansion of the undifferentiated, stem cell population of lung cancer cells (21). The results were similar to those obtained from serum-cultured A549 cells (Fig. 1B and D), suggesting that the JNK activity is required for the proliferation of A549 cells irrespective of the culture condition, at least in vitro. These results were essentially consistent with those of previous studies that demonstrated the essential role of JNK in the in vitro growth of NSCLC cells using NSCLC cell lines other than A549 (16-18).

JNK inhibition fails to inhibit the growth of established A549 tumors in vivo. Given the marked growth inhibitory effect of SP600125 on A549 cells observed in vitro, we next examined whether it has a similar inhibitory effect on the growth of A549 tumors in vivo. To this end, we implanted A549 cells subcutaneously into nude mice, and when the implanted A549 cells formed discrete subcutaneous tumors ( $7 \mathrm{~mm}$ in diameter), we treated the tumor-bearing mice by systemic, intraperitoneal administration of SP600125 (40 mg/ $\mathrm{kg}$ ) for 10 consecutive days and monitored the growth of tumors during and after the treatment. Strikingly, we observed no significant inhibition of A549 tumor growth by SP600125 under this treatment condition (Fig. 2). Although the average tumor volume of the SP600125-treated tumors was somewhat smaller than that of the control-treated tumors in this particular set of experiment (Fig. 2A), this was due to a single unique tumor that 'exceptionally' showed retarded growth in the course of SP600125 treatment, in sharp contrast to the other 9 tumors (4 SP600125-treated and 5 control-treated tumors) that all grew at a similar rate (Fig. 2B). Such a retarded growth of established A549 tumors during SP600125 treatment was never observed in any of the other sets of similar experiment, and the average tumor volume of SP600125-treated tumors was even larger (although not statistically significant) than that of the control-treated tumors in some experiments (Fig. 3A). These results clearly indicate that SP600125 is not effective at inhibiting the growth of established A549 tumors in vivo, at least under the treatment condition used in this study.

Selective elimination of the tumor-initiating cell population within established A549 tumors by JNK inhibition in vivo. Although SP600125 thus failed to control the growth of the entire bulk A549 tumors, it was nevertheless still possible that SP600125 selectively reduced the tumor-initiating cell population within the tumors, just as we demonstrated previously using glioblastoma xenografts (6). To explore this possibility, we next conducted serial transplantation experiments. Mice 

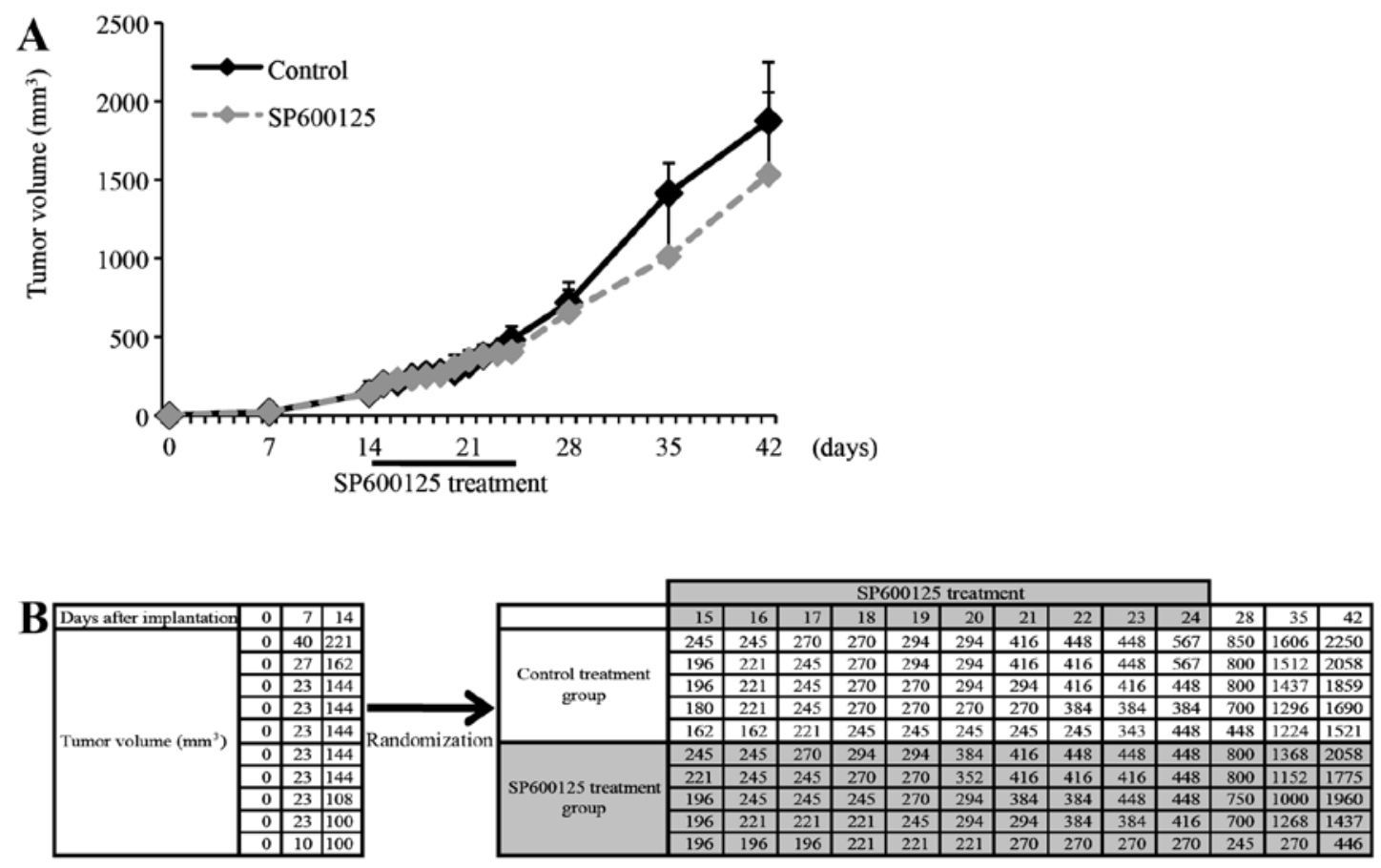

Figure 2. Failure of SP600125 to inhibit the 'bulk growth' of established A549 tumors in vivo. (A and B) Mice implanted subcutaneously with A549 cells $\left(1 \times 10^{6}\right.$ viable cells) were administered a daily intraperitoneal injection of the control vehicle or SP600125 (40 mg/kg/day) for 10 consecutive days, which started 2 weeks after the A549 cell implantation when tumor volumes were $162-245 \mathrm{~mm}^{3}$. Tumor volume at the indicated time points is presented in the graph as the mean $\pm \mathrm{SD}(\mathrm{A})$ and in the table (B).

implanted subcutaneously with A549 cells were treated by an intraperitoneal injection of SP600125 $(40 \mathrm{mg} / \mathrm{kg})$ for a consecutive 10 days when the subcutaneous tumors reached the size of 6-7 mm, similarly as in Fig. 2. The treated subcutaneous tumors were then excised, and after dissociation into single cells, serial dilutions $\left(5,2\right.$ and $\left.1 \times 10^{5}\right)$ of the dissociated cells were transplanted again subcutaneously into nude mice to initiate tumor formation. Whereas there was no significant difference in the growth of SP600125- and control-treated A549 tumors before and during the course of the treatment (Fig. 3A), tumor initiation was substantially inhibited and delayed for cells transplanted from SP600125-treated tumors as compared to those from control-treated tumors (Fig. 3B and C). We also found that the growth curve of tumors formed by transplantation of $5 \times 10^{5}$ cells from SP600125-treated tumors closely overlapped with that of tumors formed by transplantation of $1 \times 10^{5}$ cells from control-treated tumors. The results suggest that the SP600125 treatment reduced the tumor-initiating cell population within A549 tumors to $1 / 5$ in vivo.

Inhibition of tumor initiation of A549 cells by JNK inhibition in vivo. Having shown that SP600125 successfully eliminates the tumor-initiating population of A549 cells within established tumors, we next determined whether SP600125 blocks tumor initiation, albeit not bulk tumor growth, of A549 cells in vivo. To this end, we treated nude mice receiving a subcutaneous implantation of A549 cells with SP600125 using the identical treatment condition (intraperitoneal injection of $40 \mathrm{mg} / \mathrm{kg}$ SP600125 for 10 consecutive days), except that the treatment was initiated on the following day of A549 cell implantation instead of initiating SP600125 treatment after tumor forma- tion. Remarkably, the identical SP600125 treatment condition, which failed to inhibit the growth of established A549 tumors, effectively and significantly inhibited the formation of A549 tumors (Fig. 4). Thus, collectively, the results of the xenograft analyses conducted thus far suggest that systemically administered SP600125 exerts its antitumor activity against A549 cells through selective inhibition of their tumor initiation.

Essential role for the cell-intrinsic JNK activity of A549 cells in the maintenance of their tumor-initiating capacity. Since SP600125 was delivered systemically, there were two possible explanations, not mutually exclusive, for the mechanism of the antitumor activity of SP600125 observed in vivo. One was a direct mechanism, in which the cell-intrinsic JNK activity of A549 cells was required for the maintenance of their tumor-initiating capacity. The other was an indirect mechanism, in which the tumor-initiating capacity of A549 cells depended on the function(s) of other cells (i.e., cells of the host animals) sensitive to JNK inhibition. To determine the role of the 'cell-intrinsic JNK activity' in the maintenance of the tumor-initiating capacity of A549 cells, we examined the tumor-initiating capacity of A549 cells pre-treated in vitro with SP600125 before implantation into nude mice. For this xenograft analysis, A549 cells were treated with SP600125 at $20 \mu \mathrm{M}$ to inhibit JNK, for 6 days before implantation (Fig. 5A). This SP600125 treatment condition suppressed the cellular proliferation of A549 cells without causing significant reduction of their viability (Fig. 5B) as shown earlier in this study (Fig. 1). Then, after washout of SP600125, the same number $\left(5 \times 10^{5}\right.$ viable cells) of the SP600125-treated and control-treated A549 cells was implanted subcutaneously 

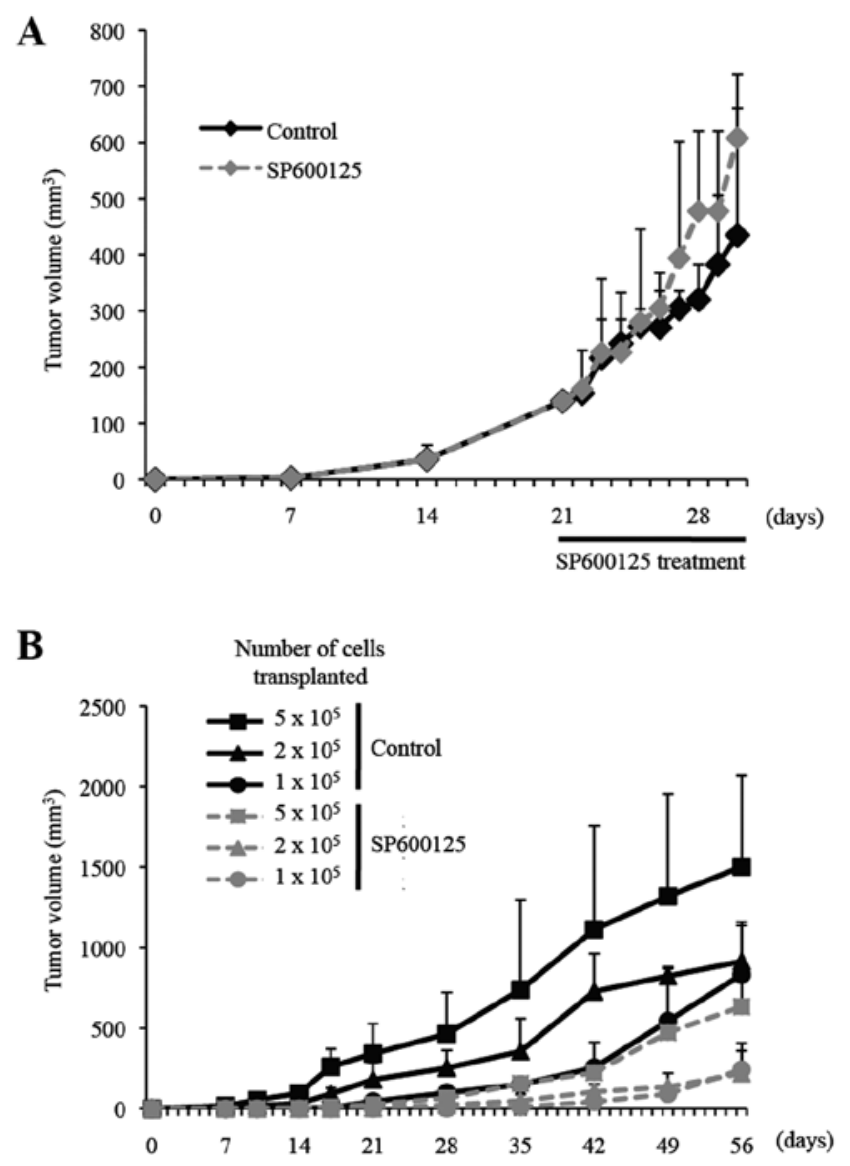

C

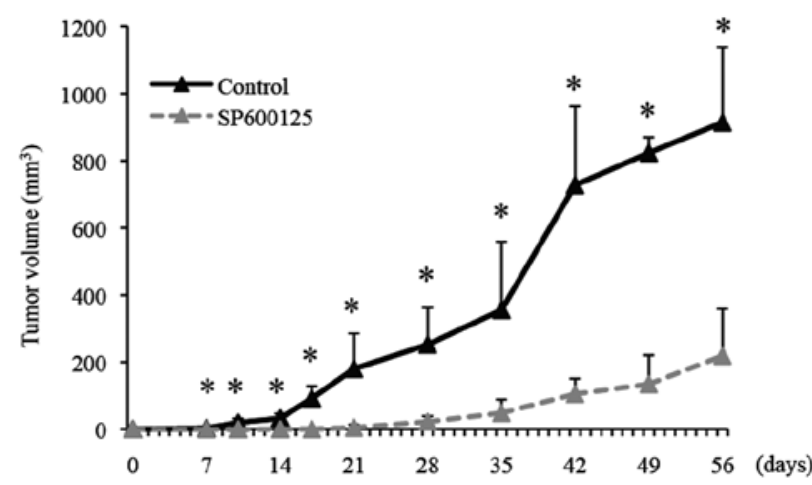

Figure 3. Selective elimination of the tumor-initiating population within established A549 tumors by SP600125 in vivo. Mice (2 mice/group) were

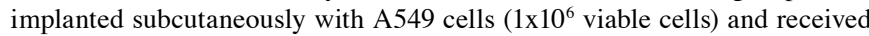
a daily intraperitoneal injection of the control vehicle or SP600125 (40 mg/kg/day) for 10 consecutive days, which started 3 weeks after the A549 cell implantation (tumor volume range at the treatment initiation, 126-144 $\mathrm{mm}^{3}$ ). On the next day of the final drug treatment, the subcutaneous tumors (primary tumors) were dissociated, and serial dilutions of the dissociated tumor cells were transplanted subcutaneously into the right flank of mice (3 mice/group). (A) The volume of the primary tumors at the indicated time points is presented in the graph as the mean \pm SD. (B) The volume of the secondary tumors formed by transplantation of the indicated numbers of viable cells from the primary tumors is presented as the mean \pm SD. For clarity, the growth curves of the secondary tumors formed by transplantation of $2 \times 10^{5}$ cells are shown again separately in $(\mathrm{C}) .{ }^{*} \mathrm{P}<0.05$.

into nude mice. The results of the xenograft analysis clearly indicated that the SP600125 pre-treatment inhibited tumor

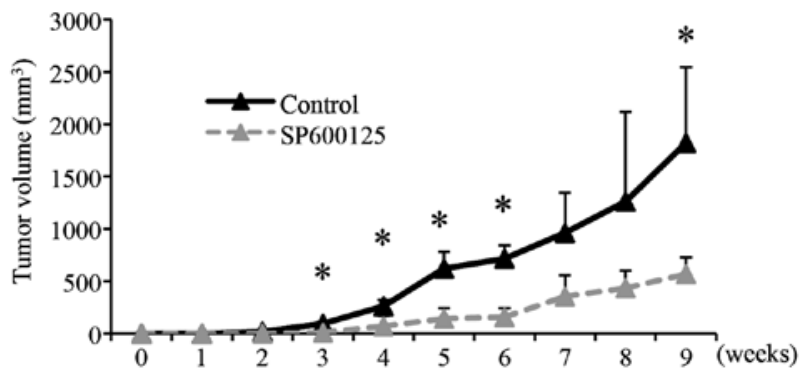

Figure 4. SP600125 inhibits tumor initiation by A549 cells in vivo. Mice implanted subcutaneously with A549 cells ( $2 \times 10^{5}$ viable cells) were administered a daily intraperitoneal injection of the control vehicle or SP600125 ( $40 \mathrm{mg} / \mathrm{kg} / \mathrm{day}$ ) for 10 consecutive days starting on the next day of implantation ( $3 \mathrm{mice} / \mathrm{group})$. Tumor volume at the indicated time points is presented in the graph as the mean $\pm \mathrm{SD}$. ${ }^{*} \mathrm{P}<0.05$.

formation by A549 cells (Fig. 5C). Notably, whereas all 3 mice receiving control-treated A549 cells developed large tumors, the three mice receiving SP600125-treated cells developed significantly smaller tumors, with one of them even surviving in a tumor-free state throughout the entire observation period (Fig. 5D). These results are in support of the idea that the cell-intrinsic activity of JNK is directly required for the maintenance of the tumor-initiating capacity of A549 cells, although they do not necessarily exclude the possibility that systemic JNK inhibition deprived A549 cells of their tumor initiation capacity through other indirect mechanisms.

Finally, to definitively establish that the cell-intrinsic $\mathrm{JNK}$ is required for the tumor-initiating capacity of A549 cells through a non-pharmacological genetic approach, we conducted knockdown experiments. To this end, we transiently transfected A549 cells with a combination of siRNAs against JNK1 and JNK2. Under the transfection condition, the knockdown of JNK1 and JNK2 was efficient, as indicated also by the reduced level of phosphorylated c-Jun (Fig. 6A). Similarly to SP600125, the JNK knockdown caused marked blockage of proliferation but not any significant reduction in the viability of the A549 cells by the time they were subjected to xenograft analysis (Fig. 6B). Subcutaneous implantation of control A549 cells and cells in which JNK1 and JNK2 were transiently knocked down revealed that the JNK knockdown significantly impaired the tumor-initiating capacity of A549 cells (Fig. 6C and D). Collectively, the results demonstrated that the cell-intrinsic JNK activity is essential for the maintenance of the tumor-initiating capacity of A549 cells.

\section{Discussion}

Since its characterization as a key molecule in the transduction of pro-apoptotic signaling $(25,26)$, JNK has long drawn considerable attention as a 'negative regulator' of tumor growth, for example, as an important mediator of cell death signals elicited by cancer therapies such as chemotherapy and radiotherapy $(27,28)$. On the other hand, accumulating evidence has now come to support the emerging idea that JNK also plays significant roles in the 'promotion' of tumor growth (3-5). Only recently, an essential role of JNK in the maintenance of the tumor-initiating capacity of glioblastoma stem cells has been documented and added as one of such 
A

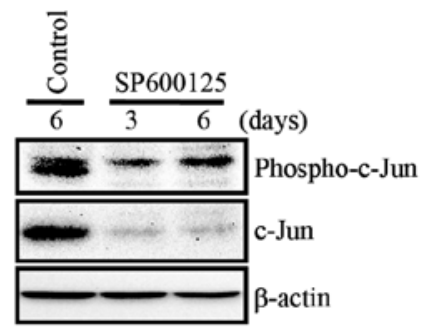

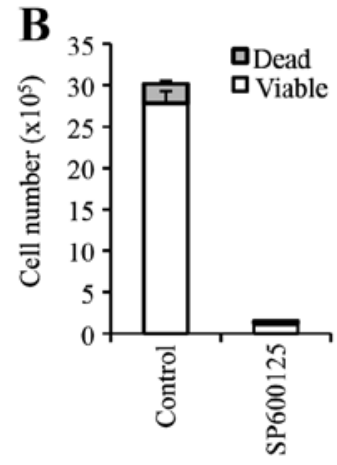

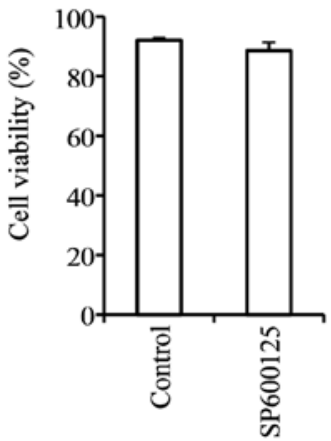

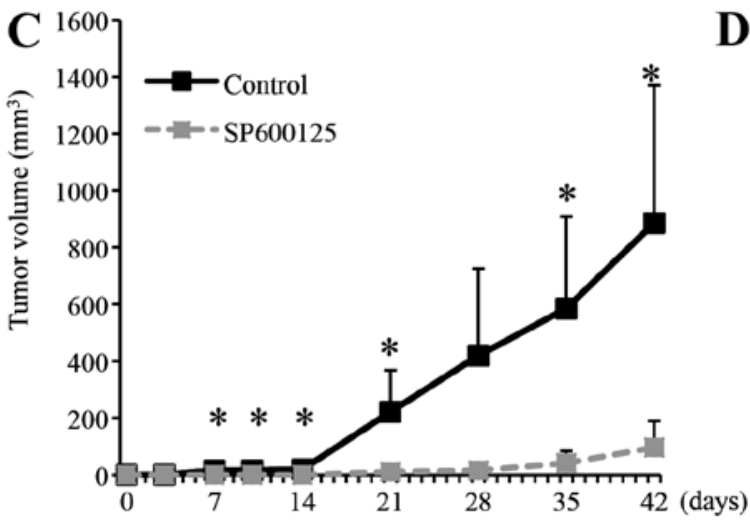

D

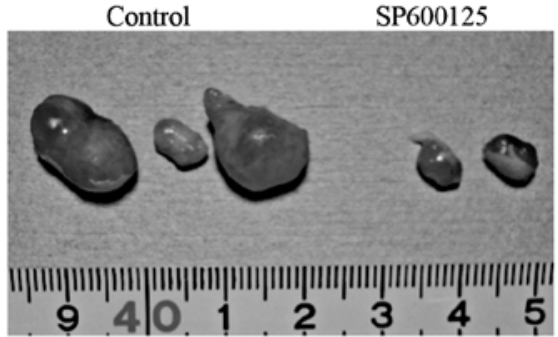
(days)

Figure 5. Pharmacologic demonstration that cell-intrinsic JNK activity is required for the maintenance of the tumor-initiating capacity of A549 cells. (A) A549 cells cultured in the absence (control) and presence of SP600125 (20 $\mu \mathrm{M})$ for the indicated time periods were examined for phospho- and total c-Jun expression by immunoblot analysis. (B and D) A549 cells cultured in the absence (control) and presence of SP600125 (20 $\mu \mathrm{M})$ for 6 days were analyzed for cell number (left) and viability (right) (B) and also subjected to subcutaneous implantation (5x10 viable cells) into the right flank of mice (3 mice/group) (C and D) (C) Tumor volume was measured at the indicated time points, and the results are presented as the means $\pm \mathrm{SD}$. ${ }^{*} \mathrm{P}<0.05$. (D) At the end of the observation period (day 42), mice were sacrificed and the subcutaneous tumors formed by control- and SP600125-treated A549 cells are shown.

'pro-tumor' roles of JNK $(6,7)$. In line with such roles of JNK, aberrant activation of JNK has been increasingly observed in human cancers (3-5). NSCLC, the major subtype of lung cancer, is among such human cancers in which activated JNK has been implicated as a positive regulator of tumor growth (16-18). However, since the cellular functions of JNK in NSCLC cells have been studied primarily in vitro (16-18), the exact role(s) of JNK in NSCLC cells in vivo still remains poorly understood. To address this issue, therefore, we conducted both in vitro and in vivo analyses of JNK functions in A549 NSCLC cells. Strikingly, in sharp contrast to the results of the in vitro analysis in which inhibition of JNK caused rapid and potent blockage of A594 cell proliferation, JNK inhibition in vivo completely failed to inhibit the growth of already established A549 tumors (Figs. 2 and 3A). However, the identical treatment condition quite efficiently deprived the tumor cells of their capacity to initiate tumors upon secondary transplantation (Fig. 3B and C), indicating that the JNK inhibitor treatment was effective and selectively targeted the tumor-initiating capacity of A549 cells while sparing their proliferative activity in vivo. The idea that JNK plays a role in the maintenance of the tumor-initiating capacity of A549 cells was further corroborated by the demonstration that the identical JNK inhibitor treatment (SP600125 $40 \mathrm{mg} / \mathrm{kg} /$ day for 10 consecutive days) that failed to inhibit the bulk tumor growth (Figs. 2 and 3A) did inhibit tumor initiation by A549 cells in vivo (Fig. 4). Apparently, the cell-intrinsic JNK activity was essential for the maintenance of the tumor-initiating capacity of A549 cells since JNK inhibition in vitro, i.e., in the absence of cells from host animals, was sufficient to deprive A549 cells of their tumor-initiating capacity (Figs. 5 and 6). Collectively, these findings give rise to and support the idea that the primary role of JNK in A549 cells in vivo, in particular in established tumors, may be to maintain their tumor-initiating capacity rather than to simply sustain cellular proliferation and survival. It needs to be emphasized here, however, that our results do not necessarily exclude the possibility that more intensified SP600125 treatment conditions would have some inhibitory effect on the growth of established A549 tumors. Nevertheless, they do dictate that we should be prudent enough not to remove JNK from the list of potential therapeutic targets even if SP600125 should prove to be ineffective at controlling bulk tumor growth in any conditions.

Another key observation we made in this study is that short-term, 'transient' inhibition of JNK was sufficient to cause 'sustained' loss of the tumor-initiating capacity of A549 cells. Except for the experiment whereby the systemic administration of SP600125 was started on the following day after A549 cell implantation (Fig. 4), the xenograft experiments examining the effect of SP600125 on the tumor-initiating capacity of A549 cells were conducted in the complete absence of SP600125 (Figs. 3B, C and 5), which is a reversible inhibitor 
A

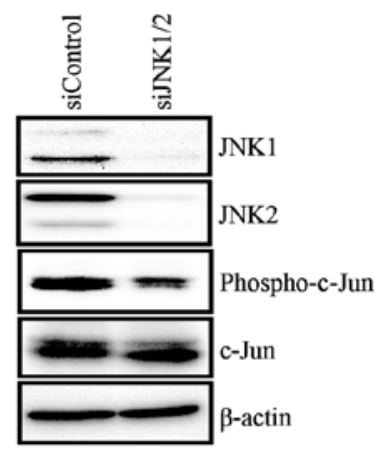

B

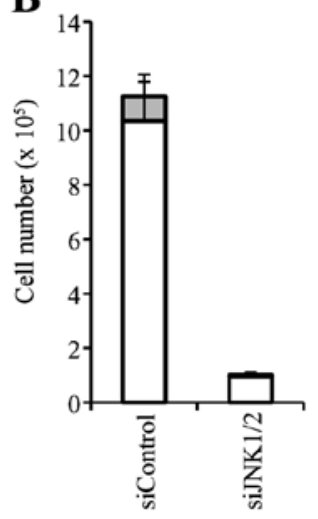

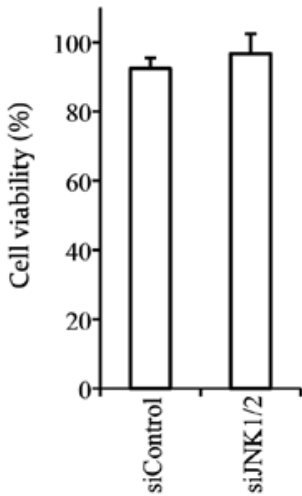

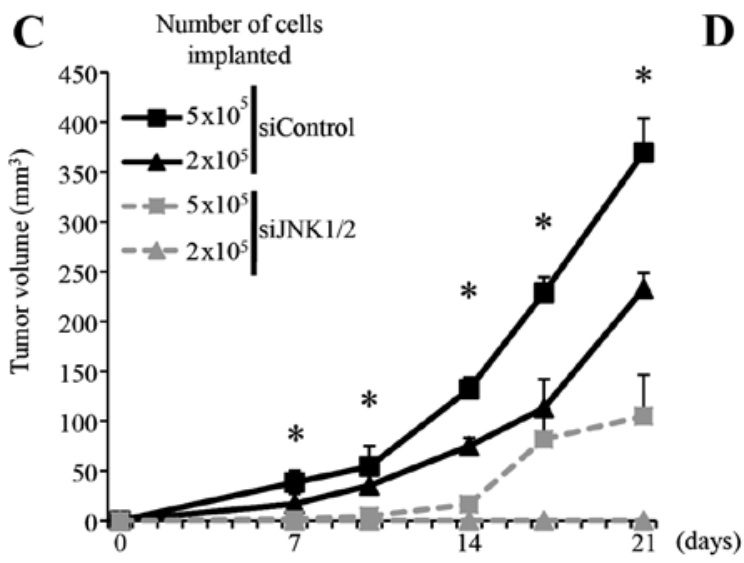

D

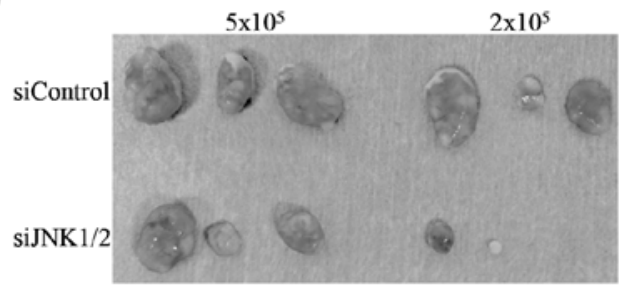

Figure 6. Genetic demonstration that cell-intrinsic JNK is required for the maintenance of the tumor-initiating capacity of A549 cells. (A and D) A549 cells were transiently transfected with siRNAs against JNK1 and JNK2 (siJNK1/2) or with a control siRNA (siControl), as detailed in Materials and methods. After 8 days, the transfected cells were subjected to immunoblot analysis for the expression of the indicated proteins (A), to cell number (left) and viability (right) analyses (B), or to xenograft analysis (C and D). For C and D, the indicated numbers of viable cells were implanted subcutaneously into the right flank of mice (3 mice/group). (C) Tumor volume was measured at the indicated time points, and the results are presented as the means \pm SD. ${ }^{*} \mathrm{P}<0.05$. (D) At the end of the observation period (day 21), mice were sacrificed and the subcutaneous tumors formed by control siRNA- and siJNK1/2-transfected cells are shown.

of JNK (24). This implies that the state of 'lost tumor-initiating capacity' is a stable one that does not require continuous inhibition of JNK, whereas continuous JNK activity was apparently required for the maintenance of the tumor-initiating capacity. In this regard, the JNK-dependent tumor-initiating capacity of A549 shares the key characteristics of what we recently described as 'stemness-associated tumor-initiating capacity (STATIC)' (5). By definition, STATIC is a type of tumor-initiating capacity regulated in close association with the stemness/differentiation of the cells most likely through an epigenetic mechanism (5). Although we need to admit that the A549 cells used in this study may not be typical bona fide CSCs since they failed to grow as non-adherent spheres in the serum-free, stem cell culture condition (data not shown), the results of the present study strongly suggest that the A549 cells analyzed in this study share with glioblastoma stem cells the core mechanism of tumor-initiating capacity, which we have just shown to be STATIC $(5,6)$. This idea is quite in good agreement with our prediction/proposal that STATIC may not always be associated with the stemness of the cells, for instance, in such a case where the regulatory mechanism governing stemness is selectively disrupted downstream of the core mechanism of STATIC that orchestrates stemness and tumor-initiating capacity (5). As such, this study may be the first instance to show that STATIC is not a property unique to CSCs, and to the best of our knowledge, is the first study to demonstrate that the cell-intrinsic JNK activity plays a pivotal role in the control of tumor-initiating capacity of cancer cells other than glioblastoma cells.

Although we clearly demonstrated using a mouse xenograft model the essential role of JNK in the maintenance of the tumor-initiating capacity of A549 cells, it still remains to be shown whether the mechanism is relevant to naturally occurring NSCLCs. In this respect, it is notable that JNK was recently shown to be essential for the development of lung tumors in a mouse model of lung adenocarcinoma driven by K-ras $(29,30)$, which is frequently activated by mutations in human lung adenocarcinomas $(31,32)$ and also in A549 cells derived from a human lung adenocarcinoma, a subtype of NSCLC $(33,34)$. Although it remains unclear how the deletion of the JNK genes (JNK1 + JNK2) contributed to the reduced formation of lung hyperplastic/neoplastic lesions and whether 'continued' as opposed to 'transient' loss of JNK (because the JNK genes were deleted genetically and therefore irreversibly in the study) is required for the suppression of lung tumor formation observed in the study (29), the findings provided 
by the study in conjunction with our current results may lend support to the idea that JNK contributes to K-ras-driven NSCLC carcinogenesis by preventing loss of tumor-initiating capacity of K-ras-transformed NSCLC cells. If this is actually the case, then JNK would be a highly promising molecular target to control recurrence of NSCLC tumors, be it either local or distant (i.e., metastasis), since any form of recurrence may involve the process of tumor initiation by tumor-initiating cells. Future studies to investigate the prevalence and significance of this JNK-regulated mechanism of tumor initiation in human NSCLCs in general, therefore, are warranted.

In conclusion, we demonstrated in the present study that JNK is specifically involved in the maintenance of the tumorinitiating capacity of A549 NSCLC cells. Of therapeutic importance, short-term, transient inhibition of JNK was sufficient to stably control the tumor-initiating capacity of A549 cells, making JNK targeting an attractive candidate as a therapeutic approach to gain long-term control over NSCLCs. Our demonstration that JNK is involved in the control of the tumor-initiating capacity of human cancer cells other than glioblastoma cells indicates that the mechanism is not unique to glioblastomas and therefore justifies further investigation of the role of JNK in the control of the tumor-initiating capacity of other human cancers.

\section{References}

1. Weston CR and Davis RJ: The JNK signal transduction pathway. Curr Opin Cell Biol 19: 142-149, 2007.

2. Kennedy NJ and Davis RJ: Role of JNK in tumor development. Cell Cycle 2: 199-201, 2003.

3. Wagner EF and Nebreda AR: Signal integration by JNK and p38 MAPK pathways in cancer development. Nat Rev Cancer 9: 537-549, 2009.

4. Chen F: JNK-induced apoptosis, compensatory growth, and cancer stem cells. Cancer Res 72: 379-386, 2012

5. Kitanaka C, Sato A and Okada M: JNK signaling in the control of the tumor-initiating capacity associated with cancer stem cells. Genes Cancer: January 22, 2013 (Epub ahead of print) doi: $10.1177 / 1947601912474892$.

6. Matsuda K, Sato A, Okada M, et al: Targeting JNK for therapeutic depletion of stem-like glioblastoma cells. Sci Rep 2: 516, 2012.

7. Yoon $\mathrm{CH}, \mathrm{Kim} \mathrm{MJ}, \mathrm{Kim} \mathrm{RK}$, et al: c-Jun N-terminal kinase has a pivotal role in the maintenance of self-renewal and tumorigenicity in glioma stem-like cells. Oncogene 31: 4655-4666, 2012.

8. Yu Y, Ramena G and Elble RC: The role of cancer stem cells in relapse of solid tumors. Front Biosci 4: 1528-1541, 2012.

9. Malik B and Nie D: Cancer stem cells and resistance to chemo and radio therapy. Front Biosci 4: 2142-2149, 2012.

10. Jemal A, Bray F, Center MM, Ferlay J, Ward E and Forman D: Global cancer statistics. CA Cancer J Clin 61: 69-90, 2011.

11. Collins LG, Haines C, Perkel R and Enck RE: Lung cancer: diagnosis and management. Am Fam Physician 75: 56-63, 2007.

12. Li JY, Wang H, May S, et al: Constitutive activation of c-Jun $\mathrm{N}$-terminal kinase correlates with histologic grade and EGFR expression in diffuse gliomas. J Neurooncol 88: 11-17, 2008.

13. Antonyak MA, Kenyon LC, Godwin AK, et al: Elevated JNK activation contributes to the pathogenesis of human brain tumors. Oncogene 21: 5038-5046, 2002.
14. Tsuiki H, Tnani M, Okamoto I, et al: Constitutively active forms of c-Jun $\mathrm{NH}_{2}$-terminal kinase are expressed in primary glial tumors. Cancer Res 63: 250-255, 2003.

15. Cui J, Han SY, Wang C, et al: c-Jun $\mathrm{NH}_{2}$-terminal kinase $2 \alpha 2$ promotes the tumorigenicity of human glioblastoma cells. Cancer Res 66: 10024-10031, 2006.

16. Khatlani TS, Wislez M, Sun M, et al: c-Jun N-terminal kinase is activated in non-small-cell lung cancer and promotes neoplastic transformation in human bronchial epithelial cells. Oncogene 26: 2658-2666, 2007.

17. Nitta RT, Del Vecchio CA, Chu AH, Mitra SS, Godwin AK and Wong AJ: The role of the c-Jun N-terminal kinase 2- $\alpha$-isoform in non-small cell lung carcinoma tumorigenesis. Oncogene 30: 234-244, 2011.

18. Lee JJ, Lee JH, Ko YG, Hong SI and Lee JS: Prevention of premature senescence requires $\mathrm{JNK}$ regulation of $\mathrm{Bcl}-2$ and reactive oxygen species. Oncogene 29: 561-575, 2010.

19. Perez EA, Hack FM, Fletcher TS and Chou TC: Modulation of intrinsic in vitro resistance to carboplatin by edatrexate in the A549 human nonsmall cell lung cancer cell line. Oncol Res 6: 151-156, 1994.

20. Eramo A, Ricci-Vitiani L, Zeuner A, et al: Chemotherapy resistance of glioblastoma stem cells. Cell Death Differ 13: 1238-1241, 2006.

21. Eramo A, Lotti F, Sette G, et al: Identification and expansion of the tumorigenic lung cancer stem cell population. Cell Death Differ 15: 504-514, 2008.

22. Ricci-Vitiani L, Lombardi DG, Pilozzi E, et al: Identification and expansion of human colon cancer-initiating cells. Nature 445: 111-115, 2007.

23. Sato A, Sunayama J, Okada M, et al: Glioma-initiating cell elimination by metformin activation of FOXO3 via AMPK. Stem Cells Transl Med 1: 811-824, 2012.

24. Bennett BL, Sasaki DT, Murray BW, et al: SP600125, an anthrapyrazolone inhibitor of Jun N-terminal kinase. Proc Natl Acad Sci USA 98: 13681-13686, 2001.

25. Xia Z, Dickens M, Raingeaud J, Davis RJ and Greenberg ME: Opposing effects of ERK and JNK-p38 MAP kinases on apoptosis. Science 270: 1326-1331, 1995.

26. Dhanasekaran DN and Reddy EP: JNK signaling in apoptosis. Oncogene 27: 6245-6251, 2008.

27. Verheij M, Ruiter GA, Zerp SF, et al: The role of the stressactivated protein kinase (SAPK/JNK) signaling pathway in radiation-induced apoptosis. Radiother Oncol 47: 225-232, 1998.

28. Fan $\mathrm{M}$ and Chambers TC: Role of mitogen-activated protein kinases in the response of tumor cells to chemotherapy. Drug Resist Updat 4: 253-267, 2001.

29. Cellurale C, Sabio G, Kennedy NJ, et al: Requirement of c-Jun $\mathrm{NH}_{2}$-terminal kinase for Ras-initiated tumor formation. Mol Cell Biol 31: 1565-1576, 2011.

30. Jackson EL, Willis N, Mercer K, et al: Analysis of lung tumor initiation and progression using conditional expression of oncogenic K-ras. Genes Dev 15: 3243-3248, 2001.

31. Vachtenheim J: Occurrence of ras mutations in human lung cancer. Minireview. Neoplasma 44: 145-149, 1997.

32. Kadara H, Kabbout M and Wistuba II: Pulmonary adenocarcinoma: a renewed entity in 2011. Respirology 17: 50-65, 2012.

33. Kurtze I, Sonnemann J and Beck JF: KRAS-mutated non-small cell lung cancer cells are responsive to either co-treatment with erlotinib or gefitinib and histone deacetylase inhibitors or single treatment with lapatinib. Oncol Rep 25: 1021-1029, 2011.

34. Okudela K, Hayashi H, Ito T, et al: K-ras gene mutation enhances motility of immortalized airway cells and lung adenocarcinoma cells via Akt activation: possible contribution to non-invasive expansion of lung adenocarcinoma. Am J Pathol 164: 91-100, 2004. 